\title{
Frecuencia de lumbalgia aguda y su tratamiento en la central de emergencias de un hospital privado
}

\author{
Acute lumbalgia frequency and treatment at a private hospital emergency plant \\ Grande-Ratti MF,* Torres-Gómez F, ${ }^{\ddagger}$ Herrera AG, ${ }^{\S}$ Crescia S, ${ }^{\circledR}$ Pollán J,» Martínez B** \\ Hospital Italiano de Buenos Aires.
}

RESUMEN. Introducción: La lumbalgia aguda (LA) es un problema en diferentes ámbitos de atención. La Central de Emergencias de Adultos (CEA) intenta brindar atención inmediata, especializada y eficaz. Nuestro objetivo fue estimar la prevalencia y describir el proceso de atención habitual. Material y métodos: Para la estimación de prevalencia, se incluyó una muestra aleatoria de 5,333 consultas admitidas en CEA de un solo hospital entre Enero y Diciembre de 2016. Se definió como numerador a los casos de LA según diagnóstico de triaje al ingreso. Una cohorte retrospectiva con todos los casos de LA se utilizó para describir estudios complementarios y conductas terapéuticas instaurados en CEA, con seguimiento ambulatorio posterior. Resultados: La lumbalgia aguda representó 4.37\% (IC 95\%: 3.83-4.95\%) de las consultas. Se incluyeron 1,096 casos de LA: edad media de $53.26 \pm 19.85$ años, 55.93\% (613) sexo femenino y escasas comorbilidades. La mediana de tiempo de atención fue de 3.28 horas y se internó $6.20 \%, 70 \%$ (778) recibió tratamiento en CEA, las vías de administración más utilizadas fueron: endovenosa (61.86\%) e intramuscular (20.62\%). Los analgésicos utilizados fueron: 55.38\% corticoides, 55.02\% AINE, 34.85\% opiáceos. Los estudios complementarios solicitados en CEA o hasta
ABSTRACT. Introduction: Acute back pain (LA) is a considerable problem in any care setting. The Emergency Department (ED) intended to provide immediate, timely and effective care. Our objective was to estimate the prevalence and describe the usual care process. Material and methods: To estimate prevalence, a random sample was included of 5,333 consultations admitted in ED at hospital XX between January and December 2016. LA cases were defined according to the diagnosis at arrival. A retrospective cohort with all LA cases was used to describe complementary studies and therapeutic behaviors established in ED or within the subsequent 30 days outpatient follow-up. Results: LA represented 4.37\% (95\% CI: 3.83-4.95\%) of consultations. A total of 1,096 cases of LA were included: mean age of $53.26 \pm 19.85$ years, $55.93 \%$ (613) female sex and few comorbidities. The median attention time was 3.28 hours and $6.20 \%$ was admitted. $70 \%$ (778) received treatment in CEA, being the most used administration routes: intravenous (61.86\%), and intramuscular (20.62\%). The analgesics used were: $55.38 \%$ corticosteroids, $55.02 \%$ NSAIDs, $34.85 \%$ opioids. The complementary studies requested during ED or until 30 days after discharge, were: $52.19 \%$ laboratory, $47.17 \%$ radiography, 28.38\% RM,

\section{Nivel de evidencia: III}

* Médica. Especialista en Medicina Familiar. Magíster en Investigación Clínica. Área de Investigación en Medicina Interna no patrocinada. https://orcid.org/0000-0001-8622-8230

${ }^{\ddagger}$ Médico. Especialista en Medicina Interna. Fellow de Perfeccionamiento Área de Investigación en Medicina Interna.

$\S$ Médica. Especialista en Medicina Familiar.

" Médica. Servicio de Clínica Médica y Central de Emergencias de Adultos.

" Médico. Jefe de Servicio de Clínica Médica.

** Médico. Servicio de Clínica Médica. Jefe de Central de Emergencias de Adultos.

Hospital Italiano de Buenos Aires. Argentina.

Correspondencia:

María Florencia Grande-Ratti

Área de Investigación en Medicina Interna. 2do piso.

Pres. Tte. Gral. Juan Domingo Perón 4190, C1199 ABH, Buenos Aires.

E-mail: maria.grande@hospitalitaliano.org.ar

Citar como: Grande-Ratti MF, Torres-Gómez F, Herrera AG, Crescia S, Pollán J, Martínez B. Frecuencia de lumbalgia aguda y su tratamiento en la central de emergencias de un hospital privado. Acta Ortop Mex. 2020; 34(6): 359-364. https://dx.doi.org/10.35366/99132 
los 30 días postegreso fueron: 52.19\% laboratorio, 47.17\% radiografía/s, 28.38\% resonancia/s, $10.77 \%$ tomografía/s, 9.12\% intervencionismo/s. Conclusiones: Este estudio aporta datos epidemiológicos sobre LA y evidencia la necesidad de fomentar el uso racional de los recursos.

Palabras clave: Dolor de la región lumbar, urgencias médicas, atención ambulatoria.

\section{Introducción}

La lumbalgia es una condición musculoesquelética que afecta a la población adulta con una prevalencia de $11.9 \%$ en el mundo. ${ }^{1}$ Se ha evidenciado un aumento durante la última década y se cree que va a seguir en aumento debido al envejecimiento de la población. ${ }^{2}$ Si bien representa un problema de salud considerable en todos los países desarrollados y es comúnmente tratado en entornos de atención primaria $^{3}$ o en la atención programada ambulatoria, ${ }^{4}$ también lo es en la internación ${ }^{5}$ y sobrecarga de las centrales de emergencias.

Debido a los altos costos en salud asociados con su abordaje y tratamiento, ${ }^{6}$ numerosos estudios se han orientado a la determinación de los factores de riesgo de aparición, de recidiva y de cronicidad de dichas manifestaciones clínicas en la población general, con la finalidad de encontrar soluciones médicas y económicas que logren reducir el impacto socioeconómico de esta patología.

Sin embargo, las herramientas clave en el diagnóstico inicial de la lumbalgia aguda (LA) siguen siendo la anamnesis $^{8,9}$ y el examen físico, ${ }^{10}$ que permiten valorar la ausencia de signos de alarma o banderas rojas y descartar procesos infecciosos o situaciones clínicas de mayor gravedad. ${ }^{11}$

En la actualidad, existen múltiples estudios complementarios a disposición para el diagnóstico: radiografía, tomografía y/o resonancia. ${ }^{2}$ En consecuencia, existe una amplia variabilidad en cuanto a la indicación de estos estudios, que dependen de múltiples factores: el lugar donde los pacientes son atendidos (disponibilidad de recursos centro-dependiente), el médico tratante (más conservadores como médicos de atención primaria o especialistas que tienden a solicitar estudios con mayor frecuencia), ${ }^{12}$ la cobertura del paciente (accesibilidad y cobertura), la demanda por parte del paciente, entre otras.

A pesar de esto, no se recomienda la realización de pruebas de imagen en pacientes con LA sin síntomas de alarma, ${ }^{13}$ ya que expone al paciente a radiación, ${ }^{14}$ sin disminuir la utilización de recursos sanitarios o dolor, ni la incapacidad a largo plazo. Sin embargo, a más de un cuarto de los pacientes con lumbalgia aguda se les solicitan estudios complementarios. ${ }^{15,16}$

En nuestro país, desconocemos cuál es la situación epidemiológica actual en el manejo de los pacientes con LA, por lo que nos propusimos estimar la prevalencia del pro-
10.77\% tomography, 9.12\% interventionism. Conclusions: This study provides epidemiological data of LA in Argentina and demonstrates the need to promote the rational use of resources.

Keywords: Low back pain, emergency medical services, ambulatory care.

blema en ámbito de urgencias y describir el proceso de atención habitual (en términos de solicitud de estudios complementarios y tratamientos indicados).

\section{Material y métodos}

Para la estimación de prevalencia, se incluyó una muestra aleatoria de 5,333 consultas admitidas en CEA entre Enero y Diciembre 2016 como denominador. Se definió como numerador a los casos de LA según el diagnóstico de triaje al ingreso.

El centro de estudio es un hospital universitario de alta complejidad (tercer nivel de atención), que ofrece servicios médicos integrales en dos sedes hospitalarias y 19 centros médicos ambulatorios periféricos. Dispone de 785 camas para internación, efectúa 47,000 egresos anuales y realiza 51,000 procedimientos quirúrgicos en sus 41 quirófanos.

La CEA atiende aproximadamente 500 consultas diarias, está constituida por cuatro áreas para la atención, diferenciados según la complejidad, la cual es definida por la condición del paciente al ingreso: cuidados críticos (Área A), cuidados intermedios (Área B), consultorios de moderada complejidad (Área C), consultorios de demanda espontánea o baja complejidad (Área D). Las Áreas C y D corresponden a casos de menor complejidad, pero representan el mayor caudal.

Toda la información relacionada con la asistencia en salud de la institución se almacena en el denominado Repositorio de Información Clínica (Clinical Data Repository [CDR]) que funciona hace más de 10 años, que tiene bases espejadas con información de-identificada (que asegura la privacidad y confidencialidad de los datos) para posibilitar el análisis secundario de la información (base para investigación y data warehouse). En este CDR también se almacenan los documentos clínicos de diversos servicios (diagnósticos, laboratorios, estudios complementarios, evoluciones ambulatorias, consultas en guardia, internaciones, atenciones no programadas, indicaciones médicas, consumos de farmacia, entre otros ejemplos). Por ende, la información de esta cohorte fue recolectada desde bases de datos secundarias administrativas de alta calidad correspondientes al sistema de atención del HIBA, integrada a la historia clínica electrónica (HCE) tradicional con un registro en una base de datos con un modelo relacional.

Los criterios de inclusión utilizados en este estudio fueron: personas $\geq 18$ años, con cualquier cobertura (prepaga, 
privada o seguro social), que hayan sido atendidos por cualquier causa en el período antes descrito en las áreas $\mathrm{C}$ o D de la CEA. Los médicos tratantes podían ser: especialistas en medicina interna o medicina familiar, en traumatología o en formación (residentes).

Los casos se definieron como LA según el diagnóstico del triaje al ingreso, recolectados en forma automática a través de términos biomédicos arborizados, es decir, asociados o ligados con un problema mayor dentro de un vocabulario controlado; aunque revisados en forma manual a través de HCE por investigadores, para la validación del verdadero caso. Se excluyeron aquéllos atendidos por politraumatismos y/o procesos infecciosos concomitantes con relación anatómica a zona lumbar.

Para describir el proceso de atención de casos que consultaron por LA (estudios complementarios y tratamientos instaurados en CEA), se construyó una cohorte retrospectiva. Los pacientes fueron seguidos desde el egreso en CEA por ambulatorio para definir los siguientes outcomes: estudios complementarios realizados hasta los 30 días postconsulta en CEA, reconsultas en CEA e internaciones no programadas hasta 60 días posteriores.

Se realizó un cálculo de tamaño muestral para estimar la prevalencia, con una frecuencia esperada de 3\% con una hemiamplitud de $2 \%$ con una confianza de $95 \%$, arrojó la necesidad de inclusión de al menos 700 consultas en guardia. Sin embargo, para responder al objeti- vo secundario, se planificó ajustar por ocho potenciales confundidores, se calculó que se requerían al menos 20 eventos por cada variable en el modelo: $20 * 8=160$ lumbalgias. Se consideró que para una frecuencia esperada de $3 \%$, para detectar al menos 160 lumbalgias se requerían incluir 5,333 consultas.

Aspectos éticos: el proyecto de investigación fue aprobado por el Comité de Ética de Protocolos de Investigación institucional (CEPI\#3076). Se garantizó el anonimato y la confidencialidad de la información obtenida, conforme a los acuerdos adoptados por las asociaciones médicas mundiales plasmadas en la declaración de Helsinki y todas sus enmiendas. No existieron riesgos potenciales para los involucrados en este estudio.

Métodos estadísticos: se presentan las variables cuantitativas de acuerdo con la distribución observada como media y desviación estándar (DE) o mediana y rango intercuartil (RIC) o percentilos 25-75. Se presentan las variables categóricas como frecuencia absoluta y frecuencia relativa (porcentaje). Las prevalencias se presentan con sus respectivos intervalos de confianza de 95\% (IC 95\%), estimados utilizando la aproximación normal.

\section{Resultados}

Dentro del período del estudio, se seleccionaron aleatoriamente 5,333 consultas por guardia de áreas D o C. La

\begin{tabular}{|c|c|c|c|}
\hline & $\begin{array}{c}\text { Lumbalgia }(\mathrm{n}=233) \\
\mathrm{n}(\%)\end{array}$ & $\begin{array}{c}\text { No lumbalgia }(\mathrm{n}=5,100) \\
\mathrm{n}(\%)\end{array}$ & $\mathrm{p}$ \\
\hline Sexo femenino & 137 (58.79) & $3,132(61.41)$ & 0.423 \\
\hline \multirow{2}{*}{ Edad } & $55.55 \pm 19.33^{*}$ & $52.72 \pm 20.81 *$ & 0.041 \\
\hline & 57 (RIC 31)** & $52(35)^{* *}$ & 0.035 \\
\hline Plan de Salud & $173(74.24)$ & $3,225(63.23)$ & 0.001 \\
\hline Forma de ingreso & & & 0.114 \\
\hline Ambulancia & $2(0.86)$ & $230(4.50)$ & \\
\hline Caminando & $231(99.14)$ & 4,864 (95.38) & \\
\hline Silla & 0 & $6(0.12)$ & \\
\hline Color de triaje & & & 0.000 \\
\hline Verde & 29 (12.44) & 1,106 (21.69) & \\
\hline Amarillo & $11(4.72)$ & 394 (7.73) & \\
\hline Rojo & 0 & $6(0.11)$ & \\
\hline Azul & 0 & $281(5.51)$ & \\
\hline Blanco & $192(82.41)$ & 3,296 (64.63) & \\
\hline Gris & $1(0.43)$ & $17(0.33)$ & \\
\hline Tipo de egreso & & & 0.058 \\
\hline Contra opinión & 0 & $39(0.77)$ & \\
\hline Alta a domicilio & 217 (93.13) & 4,467 (87.59) & \\
\hline Internación domiciliaria & $2(0.86)$ & $64(1.26)$ & \\
\hline Cancelado & 0 & $7(0.14)$ & \\
\hline Defunción & 0 & $2(0.03)$ & \\
\hline Fuga & $12(5.15)$ & $199(3.90)$ & \\
\hline Internación & $2(0.86)$ & $313(6.14)$ & \\
\hline Suspendido & 0 & $1(0.01)$ & \\
\hline Traslado a otro hospital & 0 & $8(0.16)$ & \\
\hline
\end{tabular}


Tabla 2: Características de pacientes con lumbalgia $(\mathbb{N}=1,096)$.

\begin{tabular}{|lc|}
\hline & $\mathrm{n}(\%)$ \\
\hline Edad & $53.26 \pm 19.85^{*}$ \\
& $52(33)^{* *}$ \\
\hline Sexo femenino & $613(55.93)$ \\
Cáncer y/o leucemia/linfoma/mieloma & $69(6.30)$ \\
Osteoporosis & $80(7.30)$ \\
Fractura vertebral previa & $25(2.28)$ \\
HIV & $10(0.91)$ \\
\hline HIV = virus de inmunodeficiencia humana (por sus siglas en inglés). \\
* Media \pm desviación estándar. \\
** Mediana (rango intercuartil).
\end{tabular}

prevalencia de consultas por lumbalgia aguda fue de $4.37 \%$ con IC 95\% 3.83-4.95\% (233/5,333).

Las características basales de la población según el motivo de consulta se presentan en la Tabla 1. Como puede observarse, las diferencias estadísticamente significativas $(\mathrm{p}<0.05)$ para los pacientes que consultaron por lumbalgia fueron mayoritariamente afiliados a Plan de Salud -prepaga institucional- (74 versus 63\%), tienen mayor edad (mediana 57 años versus 52 años) y fueron clasificados al ingreso por triaje con color blanco (baja complejidad para la atención con mayor frecuencia (82 versus $64 \%)$.

Dentro del grupo de casos que no consultaron por lumbalgia, los motivos de consulta más frecuentes fueron: dolor abdominal, dolor de garganta, fiebre, tos, cefalea, cuadro de vías aéreas superiores, infección del tracto urinario, entre otros.

Se atendieron 189,815 consultas en la CEA entre 01/01/2016 y 31/12/2016: 28,418 del área C, 85,797 del área D, 28,524 en demanda espontánea y 47,076 en otros sectores (consultorios de ginecología, traumatología, siquiatría, cirugía, etcétera). De un total de 142,739 (75\%) consultas de las áreas de menor complejidad (filtrado por áreas $\mathrm{C}$ y D exclusivamente), 1,159 se clasificaron como lumbalgia como motivo de consulta al ingreso del triaje. Sin embargo, se excluyeron 63 consultas por politraumatismo y/o proceso infeccioso concomitante (ejemplos: absceso lumbar, politraumatismo, fractura lumbar, dolor postraumático, punción lumbar, etcétera), restando 1,096 consultas para la descripción del proceso de atención.

En la Tabla 2 se describen las características de los pacientes con lumbalgia. Como puede observarse, se trata de pacientes jóvenes (edad media 53 años), mayoritariamente de sexo femenino (55\%), con escasas comorbilidades (< $10 \%)$.

En la Tabla 3 se describen las características de las consultas. Como puede observarse, casi la totalidad (96\%) ingresó deambulando por sus propios medios, la mediana de atención fueron 3.28 horas y $87 \%$ se fue de alta, internándose sólo $6 \%$.

Se exploraron los métodos complementarios más solicitados y los tratamientos instaurados más frecuentes, deta- llados en la Tabla 4. Tal como se evidencia, las vías de administración más frecuentes resultaron: endovenosa (61\%), intramuscular (20\%), oral/sublingual (13\%). En cuanto a los tipos de analgésicos más utilizados en guardia fueron: antiinflamatorios no esteroideos -AINE- $(55 \%)$, corticoides y opiáceos (34\%). Los estudios complementarios más solicitados (definidos como solicitados en la guardia o en forma ambulatoria hasta los 30 días postegreso) fueron: 52.19\% laboratorio, 47.17\% radiografía/s, 28.38\% resonancia/s, $10.77 \%$ tomografía/s, $9.12 \%$ intervencionismo/s.

Los casos fueron seguidos desde el egreso en guardia hasta 60 días posteriores al alta para definir los outcomes: reconsultas $38.05 \%$ (417) e internaciones $8.30 \%$ (91). Se exploraron los motivos de éstos, representando causas relacionadas: 55.64\% (232/471) y 53.85\% (49/91) respectivamente. Para más detalles ver Tabla 5.

Se exploraron los factores asociados a internaciones, resultando estadísticamente significativas: edad OR 1.04 (IC 95\%: 1.02-1.06), cáncer OR 4.19 (IC 95\%: 1.35-12.93) y fractura vertebral OR 8.92 (IC 95\%: 1.60-49.47).

Se exploraron los factores asociados con reconsultas, resultando estadísticamente significativas: internación previa 0.41 (IC 95\%: 0.19-0.85), sexo masculino OR 0.70 (IC 95\%: 0.51-0.97), edad 1.01 (IC 95\% 1.01-1.02) y osteoporosis 2.23 (IC 95\% 1.23-4.04).

\section{Discusión}

El estudio fue realizado con el fin de describir la prevalencia del problema y el proceso de atención habitualmente instaurado en nuestro medio. La prevalencia global resultó

\section{Tabla 3: Características de las consultas $(\mathbf{N}=\mathbf{1 , 0 9 6 )}$.}

\begin{tabular}{lc}
\hline & $\mathrm{n}(\%)$ \\
\hline Tiempo de atención, en minutos & $197(210.5)^{*}$ \\
Área de ingreso & \\
C & $865(78.92)$ \\
D & $231(21.08)$ \\
Día & $687(62.68)$ \\
Semana & $409(37.32)$ \\
Fin de semana o feriado & \\
Categoría de triaje & $171(15.60)$ \\
Amarillo & $533(48.63)$ \\
Verde & $391(35.68)$ \\
Blanco & $1(0.09)$ \\
Gris & \\
Forma de ingreso & $1,059(96.62)$ \\
Caminando & $2(0.18)$ \\
Silla & $35(3.19)$ \\
Ambulancia & \\
Tipo de egreso & $956(87.23)$ \\
Alta & $68(6.20)$ \\
Internación & $11(1.00)$ \\
Internación domiciliaria & $57(5.20)$ \\
Fuga, cancelación, suspensión & $4(0.36)$ \\
Contra opinión & \\
* Mediana (rango intercuartil). & \\
\hline
\end{tabular}


Tabla 4: Estudios complementarios y tratamientos instaurados $(\mathrm{N}=1,096)$.

\begin{tabular}{|c|c|}
\hline & n (\%) \\
\hline $\begin{array}{l}\text { Realización de laboratorio } \\
\text { (por guardia }+30 \text { días) }\end{array}$ & $572(52.19)$ \\
\hline $\begin{array}{l}\text { Número de prácticas } \\
\text { (definida como laboratorio o imágenes) }\end{array}$ & $4(56)^{*}$ \\
\hline $\begin{array}{l}\text { Realización de radiografías } \\
\text { (por guardia }+30 \text { días) }\end{array}$ & $517(47.17)$ \\
\hline $\begin{array}{l}\text { Realización de intervencionismo } \\
\quad \text { (por guardia }+30 \text { días) }\end{array}$ & $100(9.12)$ \\
\hline $\begin{array}{l}\text { Realización de tomografías } \\
\text { (por guardia }+30 \text { días) }\end{array}$ & $118(10.77)$ \\
\hline $\begin{array}{l}\text { Realización de resonancias } \\
\text { (por guardia }+30 \text { días) }\end{array}$ & $311(28.38)$ \\
\hline Recibió medicación vía oral & 149 (13.59) \\
\hline Recibió medicación vía intramuscular & $226(20.62)$ \\
\hline Recibió medicación vía endovenosa & $678(61.86)$ \\
\hline $\begin{array}{l}\text { Recibió medicación por otra vía de } \\
\text { administración }\end{array}$ & $101(9.22)$ \\
\hline Recibió AINE & $603(55.02)$ \\
\hline Recibió opiáceos & $382(34.85)$ \\
\hline Recibió corticoides & $607(55.38)$ \\
\hline
\end{tabular}

en $4.37 \%$ (IC 95\% 3.83-4.95\%), un poco inferior comparado con los reportes bibliográficos. ${ }^{1}$ Algunos puntos de interés a considerar del estudio fueron: 1) si bien es unicéntrico, se trata de un hospital de alta complejidad y con gran caudal de pacientes, aportando en un año 1,096 casos de lumbalgia en guardia, dentro de los sectores de atención de baja complejidad; mientras que otros estudios multicéntricos incluyeron 559 casos en tres centros; ${ }^{17}$ 2) el amplio tamaño muestral permite estimar los resultados con precisión.

Sin embargo, la importancia clínica del trabajo radica en la descripción del proceso de atención habitual en nuestro medio, en términos de estudios complementarios solicitados y tratamientos instaurados. De hecho, el grupo de trabajo Choosing Wisely Canada publicó recientemente una lista de 52 pruebas, procedimientos y tratamientos en emergencias, cuyo valor para el cuidado del paciente fue cuestionado. ${ }^{18}$ Luego de una votación de expertos (basada en capacidad de acción, efectividad, seguridad, carga económica y frecuencia de uso), una de las cinco prioridades resultó ser «no solicitar imágenes de la columna lumbosacra en pacientes con dolor lumbar no traumático que no tienen banderas rojas y/o indicadores patológicos». Nuestro estudio arrojó que los estudios complementarios más solicitados (definido como en la guardia o hasta los 30 días postegreso) fueron: 52.19\% laboratorio, 47.17\% radiografía/s, 28.38\% resonancia/s, $10.77 \%$ tomografía/s, 9.12\% intervencionismo/s. Sólo en 10-20\% de las lumbalgias agudas y en 10-45\% de las crónicas el síntoma se puede atribuir a una lesión anatómica precisa. ${ }^{19}$ Por tanto, la mayoría de las veces el manejo terapéutico no puede ser sino sintomático. La prescripción de AINE para el tratamiento del dolor lumbar ha ido aumentando. ${ }^{20}$
En nuestro estudio, los tipos de analgésicos más utilizados en guardia: 55.02\% recibieron AINE, 55.38\% corticoides, 34.85\% opiáceos.

Esta evidencia conlleva a reflexionar sobre la atención médica de urgencia, que constituye un punto crucial para la calidad asistencial en los sistemas de salud. Algunos estudios exploraron el método clínico para mejorar el uso de recursos y los costos, con la intención de identificar el uso de exámenes complementarios y medicamentos, sus costos y relación con la morbilidad observada. ${ }^{21}$ Varias consideraciones se desprenden, principalmente se requieren mayores esfuerzos para fomentar el uso racional de medicación y estudios complementarios en guardia. Por un lado, se podría pensar en mejorar la continuidad y el acceso a atención primaria o el contacto con médico de cabecera en ámbito ambulatorio, que podría significar la consecuente reducción de los sistemas de emergencias. ${ }^{22}$ Un dato de interés fue que $18.16 \%$ de los casos tuvieron consulta con médico de cabecera dentro de los 60 días posteriores a la consulta en guardia dentro de nuestra cohorte, mientras que 37.50\% tuvieron consulta con traumatología. Sin embargo, según la bibliografía, 90\% de los pacientes pueden ser controlados por el médico de atención primaria y solamente $10 \%$ tiene que ser derivado a especialistas de otro nivel de atención. Por otro lado, durante los últimos años se está produciendo un importante incremento en la utilización de los servicios de urgencias con la consiguiente sobresaturación. ${ }^{23}$ Por eso, otra aproximación pendiente sería explorar cuáles son los determinantes de las utilización de los servicios de urgencias. $^{21,24}$

Por último, este estudio representa un diagnóstico situacional de la lumbalgia como motivo de consulta a central de emergencia, que podría ser información útil para programar un futuro proyecto de gestión, diseñar un plan de acción estratégico, construcción de indicadores de calidad específicos y propuestas de mejora de los componentes de atención.

\begin{tabular}{|lc|}
\hline \multicolumn{2}{|c}{ Tabla 5: Descripción del proceso de seguimiento } \\
de los pacientes (N = 1,096).
\end{tabular}




\section{Agradecimiento}

Los autores agradecen a la Dra. Karin Kopitowski por el apoyo general como Jefa de Servicio de Medicina Familiar en este proyecto.

Referencias

1. Hoy D, Bain C, Williams G, March L, Brooks P, Blyth F, et al. A systematic review of the global prevalence of low back pain. Arthritis Rheum. 2012; 64(6): 2028-37. doi: 10.1002/art.34347.

2. Allegri M, Montella S, Salici F, Valente A, Marchesini M, Compagnone $C$, et al. Mechanisms of low back pain: a guide for diagnosis and therapy. F1000Res. 2016; 5. doi: 10.12688/f1000research.8105.2.

3. Aguilera A, Herrera A. Lumbalgía: una dolencia muy popular y a la vez desconocida. Comunidad y Salud. 2013; 11(2): 80-9.

4. Hoy D, March L, Brooks P, Blyth F, Woolf A, Bain C, et al. The global burden of low back pain: estimates from the Global Burden of Disease 2010 study. Ann Rheum Dis. 2014; 73(6): 968-74. doi: 10.1136/ annrheumdis-2013-204428.

5. Soto-Padilla M, Espinosa-Mendoza RL, Sandoval-García JP, GómezGarcía F. Frecuencia de lumbalgia y su tratamiento en un hospital privado de la Ciudad de México. Acta Ortop Mex. 2015; 29(1): 40-5.

6. Dagenais S, Caro J, Haldeman S. A systematic review of low back pain cost of illness studies in the United States and internationally. Spine J. 2008; 8(1): 8-20. doi: 10.1016/j.spinee.2007.10.005.

7. Poiraudeau S, Lefevre Colau MM, Fayad F, Rannou F, Revel M. Lumbalgias. EMC - Aparato Locomotor. 2004; 37(4): 1-17. https:// doi.org/10.1016/S1286-935X(04)70536-5. Disponible en: http://www. sciencedirect.com/science/article/pii/S1286935X04705365

8. Skovron ML, Szpalski M, Nordin M, Melot C, Cukier D. Sociocultural factors and back pain. A population-based study in Belgian adults. Spine (Phila Pa 1976). 1994; 19(2): 129-37.

9. Chou R, Qaseem A, Owens DK, Shekelle P; Clinical Guidelines Committee of the American College of Physicians. Diagnostic imaging for low back pain: advice for high-value health care from the American College of Physicians. Ann Intern Med. 2011; 154(3): 1819. doi: 10.7326/0003-4819-154-3-201102010-00008.

10. Borczuk P. An evidence-based approach to the evaluation and treatment of low back pain in the emergency department. Emerg Med Pract. 2013; 15(7): 1-23; Quiz 23-4.

11. Pérez Irazusta I, Alcorta Michelena I, Aguirre Lejarcegui G, Aristegi Racero G, Caso Martínez J, Esquisabel Martinez R, et al. Guía de práctica clínica sobre lumbalgia Osakidetza. GPC 2007/1. Vitoria-Gasteiz. Disponible en: https://www.osakidetza.euskadi.eus/ contenidos/informacion/osk_publicaciones/eu_argital/adjuntos/gidak/ guiaLumbalgia.pdf

12. Maher C, Underwood M, Buchbinder R. Non-specific low back pain. Lancet. 2017; 389(10070): 736-47. doi: 10.1016/S01406736(16)30970-9.

13. Chou R, Fu R, Carrino JA, Deyo RA. Imaging strategies for low-back pain: systematic review and meta-analysis. Lancet. 2009; 373(9662): 463-72. doi: 10.1016/S0140-6736(09)60172-0.
14. Berrington de González A, Darby S. Risk of cancer from diagnostic X-rays: estimates for the UK and 14 other countries. Lancet. 2004; 363(9406): 345-351.

15. Chou R, Qaseem A, Snow V, Casey D, Cross JT Jr, Shekelle P, et al. Diagnosis and treatment of low back pain: a joint clinical practice guideline from the American College of Physicians and the American Pain Society. Ann Intern Med. 2007; 147(7): 478-91. doi: 10.7326/0003-4819-147-7-200710020-00006.

16. Morgan DJ, Dhruva SS, Wright SM, Korenstein D. 2016 update on medical overuse: a systematic review. JAMA Intern Med. 2016; 176(11): 1687-92. doi: 10.1001/jamainternmed.2016.5381.

17. Kohns DJ, Haig AJ, Uren B, Thompson J, Muraglia KA, Loar S, et al. Clinical predictors of the medical interventions provided to patients with low back pain in the emergency department. J Back Musculoskelet Rehabil. 2018; 31(1): 197-204. doi: 10.3233/BMR170806.

18. Cheng AHY, Campbell S, Chartier LB, Goddard T, Magee K, McEwen J, et al. Choosing Wisely Canada ${ }^{\circledR}$ : five tests, procedures and treatments to question in Emergency Medicine. CJEM. 2017; 19(S2): S9-S17. doi: 10.1017/cem.2017.1.

19. Bernard TN Jr, Kirkaldy-Willis WH. Recognizing specific characteristics of nonspecific low back pain. Clin Orthop Relat Res. 1987; (217): 266-820.

20. Sáez-Jiménez R, Bonis J. Estudio descriptivo sobre el uso de antiinflamatorios no esteroideos por vía intramuscular para el tratamiento de la lumbalgia aguda en las consultas de Atención Primaria en España durante 2002-2011. Rev Clin Med Fam. 2015; 8(2): 103-9.

21. Díaz Novás J, Calles Calviño A, Roldán de la Paz I, Chea Ochoa D. Las urgencias en la atención primaria: uso de exámenes complementarios y medicamentos. Rev Cubana Med Gen Integr [Internet]. 2008; 24(3). Disponible en: http://scielo.sld.cu/scielo. php?script=sci_arttext\&pid=S0864-21252008000300003\&lng=es

22. LaCalle E, Rabin E. Frequent users of emergency departments: the myths, the data, and the policy implications. Ann Emerg Med. 2010; 56(1): 42-8. doi: 10.1016/j.annemergmed.2010.01.032.

23. Giunta DH, Pedretti AS, Elizondo CM, Grande Ratti MF, González Bernaldo de Quiros F, Waisman GD, et al. Descripción de las características del fenómeno Crowding en la Central de Emergencia de Adultos, en un hospital universitario de alta complejidad: estudio de cohorte retrospectiva. Rev Med Chil. 2017; 145(5): 557-63. doi: 10.4067/S0034-98872017000500001.

24. Sarría Santamera A, Prado Galbarro J, Quintana Díaz M, Martínez Virto A. Determinantes de la utilización de los servicios de urgencias en Espino. Asunción Primaria. 2015; 47(6): 386-8. https://doi.org/10.1016/j.aprim.2014.09.011. Disponible en: http://www.sciencedirect.com/science/article/pii/ S0212656714003941?via\%3Dihub

Fuentes de financiación: Esta investigación no fue financiada.

Conflicto de intereses: Los autores declaran no tener conflicto de interes 